Research Article

\title{
Graphs Whose Edge Set Can Be Partitioned into Maximum Matchings
}

\author{
Niraj Khare \\ Department of Mathematics, The Ohio State University, Columbus, OH 43210, USA \\ Correspondence should be addressed to Niraj Khare; khare.9@osu.edu
}

Received 17 October 2012; Accepted 19 November 2012

Academic Editors: A. V. Kelarev and B. Wu

Copyright (C) 2013 Niraj Khare. This is an open access article distributed under the Creative Commons Attribution License, which permits unrestricted use, distribution, and reproduction in any medium, provided the original work is properly cited.

This paper provides structural characterization of simple graphs whose edge set can be partitioned into maximum matchings. We use Vizing's classification of simple graphs based on edge chromatic index.

\section{Introduction}

By a simple graph, we shall mean a graph with no loop and no multiple edges. We will only consider simple graphs with no isolated vertex. We first fix some notations. For a graph $G, E(G)$, and $V(G)$ would denote the edge set and the vertex set of $G$, respectively. $\Delta(G), v(G)$, and $\chi^{\prime}(G)$ would denote the maximum degree of any vertex in $G$, the size of a maximum matching in $G$ and the edge chromatic index of $G$, respectively. For $x \in V(G), \operatorname{deg}_{G}(x)$ would denote the degree of the vertex $x$ and $G \backslash x$ would denote the induced subgraph on $V(G) \backslash\{x\}$.

We now consider simple graphs whose edge set can be partitioned into maximum matchings. Complete graphs and even cycles are some of the examples but there are numerous other examples too. For instance, consider the graph in Figure 1.

Vizing's celebrated theorem states that $\chi^{\prime}(G) \leq \Delta(G)+1$ for a simple graph $G$. The definition of the edge chromatic index implies that $\chi^{\prime}(G) \geq \Delta(G)$. Therefore Vizing classified simple graphs as follows: a simple graph $G$ is in class I if and only if $\chi^{\prime}(G)=\Delta(G)$ and a simple graph $G$ is in class II if and only if $\chi^{\prime}(G)=\Delta(G)+1$. There is no structural characterization yet known for the graphs in class I or in class II. It is NP-complete to determine whether a simple graph is in Class I or Class II (see [1]). But under certain restrictions structural characterization of class I and class II graphs has been achieved. It is also known that all planar graphs with maximum degree at least seven are in Class I (see $[2,3])$. Another interesting result concerns itself with relative cardinality of Class I and Class II (see [4]). We will characterize Class I and class II graphs whose edge set can be partitioned into maximum matchings.

\section{Results}

Our main aim in this paper is to prove the following results.

Theorem 1. Let $G$ be a simple graph such that $v(G) \geq 2, \Delta(G) \geq$ 2 , and $|E(G)|=\Delta(G) v(G)+\lfloor v(G) /\lceil\Delta(G) / 2\rceil\rfloor\lfloor\Delta(G) / 2\rfloor$. $G$ is a unique graph up to isomorphism if and only if $\lceil\Delta(G) / 2\rceil$ divides $v(G)$.

Theorem 2. If $G$ is a Class II graph and $E(G)$ has a partition into maximum matchings, then $\Delta(G)$ is even and $G$ is the graph with exactly $2 \nu(G) / \Delta(G)$ components each isomorphic to $K_{\Delta(G)+1}$, the complete graph of order $\Delta(G)+1$.

Theorem 3. If $G$ is a Class I graph and $E(G)$ can be partitioned into maximum matchings, then $E(G)$ has a partition into subgraphs that are either $K_{1, \Delta(G)}$ or a factor critical graph $H$ such that $E(H)$ can also be partitioned into maximum matchings and $\chi^{\prime}(H)=\chi^{\prime}(G)$.

\section{Preliminaries}

We first establish some basic results that will be extremely useful in the next section. We will be borrowing some ideas and results discussed in [5]. The following definition is in [5]. 


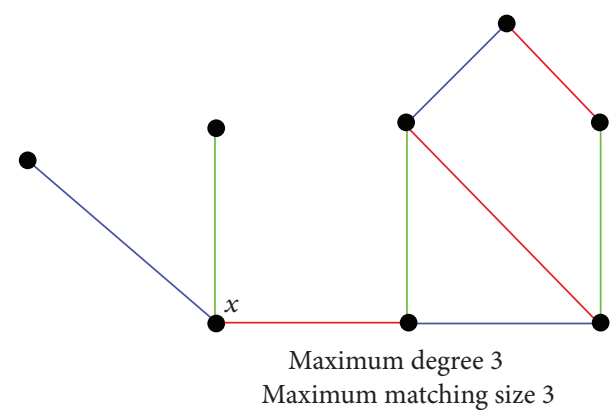

FIGURE 1: A graph whose edge set can be partitioned into maximum matchings.

Definition 4. Let $d, m$ be positive integers and $G$ be a simple graph with $\Delta(G)<d$ and $v(G)<m$. $G \in \mathscr{F}(d, m)$ if and only if for any simple graph $G^{\prime}$ such that $\left|E\left(G^{\prime}\right)\right|>|E(G)|$ and $G$ is a subgraph of $G^{\prime}$, either $\Delta\left(G^{\prime}\right) \geq d$ or $v\left(G^{\prime}\right) \geq m$.

Theorem 5. For a simple graph $G$, if $v:=v(G)$ and $\Delta:=$ $\Delta(G)$, then

$$
\begin{gathered}
|E(G)| \leq \Delta v+\left\lfloor\frac{v}{\lceil\Delta / 2\rceil}\right\rfloor\left\lfloor\frac{\Delta}{2}\right\rfloor \\
|E(G)| \leq v\left(\Delta+\frac{\lfloor\Delta / 2\rfloor}{\lceil\Delta / 2\rceil}\right) .
\end{gathered}
$$

Proof. It is obvious that for each graph $G$ with $\Delta(G)=\Delta$ and $v(G)=v$ there exists a maximal graph $G^{\prime}$ such that $v\left(G^{\prime}\right)=v$, $\Delta\left(G^{\prime}\right)=\Delta,\left|E\left(G^{\prime}\right)\right| \geq|E(G)|$ and $G^{\prime} \in \mathscr{F}(\Delta+1, v+1)$. Note that a maximal graph such as $G^{\prime}$ can be constructed by adding edges or new vertices and edges to $G$. The upper bound for $|E(H)|$ obtained in [5] implies that for any $H \in \mathscr{F}(d, m)$, $|E(H)| \leq(d-1)(m-1)+\lfloor(m-1) /\lceil(d-1) / 2\rceil\rfloor(d-1) / 2\rfloor$. Therefore,

$$
\begin{aligned}
\left|E\left(G^{\prime}\right)\right| & \left.\leq \Delta v+\left\lfloor\frac{v}{\lceil\Delta / 2\rceil}\right\rfloor \frac{\Delta}{2}\right\rfloor \\
& \leq v\left(\Delta+\frac{\lfloor\Delta / 2\rfloor}{\lceil\Delta / 2\rceil}\right) .
\end{aligned}
$$

Note that the above bound can also be inferred from [6]. However, the method used in [6] is more involved and it does not help in ascertaining uniqueness of the graphs achieving the edge bound (1) for given $\Delta$ and $v$ values, that is, Theorem 1. Our first goal is to prove Theorem 1. We next define a factor-critical graph and state the Gallai's lemma that is crucial to the following discussion. An elegant proof of the Gallai's lemma can be found in [7].

Definition 6. A simple, connected graph $G$ is called factorcritical if and only if $G \backslash x$ has a perfect matching for all $x \in$ $V(G)$.

Lemma 7 (Gallai). Let $G$ be a simple, connected graph. If $v(G \backslash$ $x)=v(G)$ for all $x \in V(G)$, then $G$ is a factor-critical graph.
We will consider graphs with no isolated vertex only. Now we consider those graphs that attain the edge bound and analyze under what conditions these graphs are unique. We first consider some trivial cases.

Remark 8. For $\Delta=1$, the graph that attains the edge bound (1) consists of $v$ components where each component is $K_{2}$. For $v=1$ and $\Delta>3$, the unique graph that attains the edge bound ( 1 ) is $K_{1, \Delta}$. For $v=1$ and $\Delta=2$ the unique graph that attains the edge bound is $K_{3}$. But there are two graphs $G$ that satisfy $\Delta(G) \leq 3, v(G)=1$ and attain the edge bound $|E(G)|=3$. These graphs are $K_{3}$ and $K_{1,3}$.

We next consider all cases involving $\Delta \geq 2$ and $v \geq 2$.

3.1. Unique Graph $\mathscr{C}$. Let $\Delta \geq 2$ be a positive integer. We consider simple graphs $G$ with no isolated vertex such that

$$
\begin{gathered}
v(G)=\left\lceil\frac{\Delta}{2}\right\rceil, \\
\Delta(G)=\Delta, \\
|E(G)|=\left\lfloor\frac{(2(\lceil\Delta / 2\rceil)+1)(\Delta)}{2}\right\rfloor=\left(\Delta+\frac{\lfloor\Delta / 2\rfloor}{\lceil\Delta / 2\rceil}\right)\left\lceil\frac{\Delta}{2}\right\rceil .
\end{gathered}
$$

Note that the last equation ensures that $|E(G)|$ attains the maximum edge bound given by (1). We will now construct a graph $\mathscr{C}$ satisfying (4) as follows.

Case (I). Let $\Delta$ be an even integer. In this case, let $\mathscr{C}=$ $K_{\Delta+1}$.

Case (II). Let $\Delta=2 j-1$ for some $j \geq 2$. In this case, let $\mathscr{E}$ be the graph obtained from $K_{2 j}$ by removing a maximum matching. To obtain $\mathscr{C}$, we connect any $2 j-1$ of the vertices of $\mathscr{E}$ to a new vertex, $v \notin V(\mathscr{E})$.

We next prove that $\mathscr{C}$ is the unique graph satisfying (4).

Proposition 9. Let $\Delta \geq 2$ and $\mathscr{C}$ be the simple graph described above. If $G$ is a simple graph satisfying (4) then
(a) $v(G \backslash x)=v(G)$ for all $x \in V(G)$,
(b) $G$ is connected,
(c) $G \simeq \mathscr{C}$.

Proof. Let $G$ be a graph satisfying the conditions of the proposition.

Proof of (a). If the statement (a) is false then there exists a vertex $x \in V(G)$ such that $v(G \backslash x)<v(G)$. As at most one edge can cover $x$ in any maximum matching, we have $v(G \backslash x)=v(G)-1$. Therefore,

$$
\begin{aligned}
|E(G)| \leq & |E(G \backslash x)|+\Delta(G \backslash x) \\
\leq & \left(\Delta(G \backslash x)+\frac{\lfloor\Delta(G \backslash x) / 2\rfloor}{\lceil\Delta(G \backslash x) / 2\rceil}\right) v(G \backslash x) \\
& +\Delta(G \backslash x)[\text { by }(2)]
\end{aligned}
$$


The above expression is a nondecreasing function of $\Delta(G \backslash x)$ for a fixed $v(G \backslash x)$. Since $\Delta \geq \Delta(G \backslash x)$, we have

$$
\begin{aligned}
|E(G)| & \leq\left(\Delta+\frac{\lfloor\Delta / 2\rfloor}{\lceil\Delta / 2\rceil}\right) v(G \backslash x)+\Delta \\
& =\left(\Delta+\frac{\lfloor\Delta / 2\rfloor}{\lceil\Delta / 2\rceil}\right)(v-1)+\Delta \\
& =\left(\Delta+\frac{\lfloor\Delta / 2\rfloor}{\lceil\Delta / 2\rceil}\right) v-\frac{\lfloor\Delta / 2\rfloor}{\lceil\Delta / 2\rceil} .
\end{aligned}
$$

Therefore by assumption and the above equation, we have $|E(G)| \leq|E(G)|-\lfloor\Delta / 2\rfloor /\lceil\Delta / 2\rceil$. But $0<\lfloor\Delta / 2\rfloor /\lceil\Delta / 2\rceil$, since $\Delta \geq 2$. Hence the statement (a) holds.

Proof of $(b)$. On the contrary assume that $G$ is not connected. Let $\mathscr{C}_{1}$ be a component of $G$. Then $1 \leq v\left(\mathscr{C}_{1}\right)<v(G)=$ $\lceil\Delta / 2\rceil$ as $G$ has no isolated vertex and $G$ is not connected by assumption. By statement (a) and Gallai's Lemma (Lemma 7), $\mathscr{C}_{1}$ is a factor-critical component. Therefore, $\left|V\left(\mathscr{C}_{1}\right)\right|=$ $2 v\left(\mathscr{C}_{1}\right)+1$. So,

$$
\left|E\left(\mathscr{C}_{1}\right)\right| \leq\left(2 v\left(\mathscr{C}_{1}\right)+1\right) \frac{\Delta\left(\mathscr{C}_{1}\right)}{2} \leq\left(2 v\left(\mathscr{C}_{1}\right)+1\right) v\left(\mathscr{C}_{1}\right) .
$$

The above inequality implies that

$$
\begin{aligned}
\frac{\left|E\left(\mathscr{C}_{1}\right)\right|}{v\left(\mathscr{C}_{1}\right)} & \leq 2 v\left(\mathscr{C}_{1}\right)+1 \\
& \leq 2\left(\left\lceil\frac{\Delta}{2}\right\rceil-1\right)+1 \\
& <\Delta+\frac{\lfloor\Delta / 2\rfloor}{\lceil\Delta / 2\rceil} \quad\left[\text { as for } \Delta \geq 2,2\left(\left\lceil\frac{\Delta}{2}\right\rceil-\frac{\Delta}{2}\right)\right. \\
& \left.\quad-1 \leq 0<\frac{\lfloor\Delta / 2\rfloor}{\lceil\Delta / 2\rceil}\right]
\end{aligned}
$$

So there is a component $\mathscr{C}_{2}$ of $G$ such that $\left(\left|E\left(\mathscr{C}_{2}\right)\right| / \nu\left(\mathscr{C}_{2}\right)\right)>$ $\Delta+(\lfloor\Delta / 2\rfloor /\lceil\Delta / 2\rceil)$ as $(|E(G)| / v(G))=\Delta+(\lfloor\Delta / 2\rfloor /\lceil\Delta / 2\rceil)$. But the (2) demands that $\left(\left|E\left(\mathscr{C}_{2}\right)\right| / \nu\left(\mathscr{C}_{2}\right)\right) \leq \Delta+(\lfloor\Delta / 2\rfloor /\lceil\Delta / 2\rceil)$. The contradiction implies that the statement (b) holds.

Proof of (c). Since the statements (a) and (b) hold for $G, G$ is factor-critical by Gallai's Lemma (Lemma 7). As $v(G)=$ $\lceil\Delta / 2\rceil$, we have $|V(G)|=2(\lceil\Delta / 2\rceil)+1$. We consider following two cases.

If $\Delta$ is even then $G$ is a connected graph with $2(\lceil\Delta / 2\rceil)+$ $1=\Delta+1$ vertices and $|E(G)|=(\Delta+(\lfloor\Delta / 2\rfloor /\lceil\Delta / 2\rceil))\lceil\Delta / 2\rceil=$ $((\Delta+1) \Delta / 2)$. Therefore, $\operatorname{deg}_{G}(x)=\Delta$ for all $x \in V(G)$. Hence $G$ is $K_{\Delta+1}$, the complete graph on $\Delta+1$ vertices. So $G \simeq \mathscr{C}$.

If $\Delta$ is odd. Let $\Delta=2 j-1$ for some $j \geq 2$. Then $v(G)=$ $\lceil\Delta / 2\rceil=j,|V(G)|=2 v(G)+1=2 j+1$ and $|E(G)|=(\Delta+$ $(\lfloor\Delta / 2\rfloor /\lceil\Delta / 2\rceil))\lceil\Delta / 2\rceil=(2 j-1) j+j-1$. So

$$
\sum_{x \in V(G)} \operatorname{deg}_{G}(x)=2 j(2 j-1)+2 j-2 .
$$

Therefore there is a unique vertex $v \in V(G)$ of degree $2 j-$ 2. Hence there is a vertex $u$ in $V(G)$ which is not a neighbor of $v$. Consequently $G \backslash u$ is a regular graph of degree $2 j-2$ on $2 j$ vertices and hence its complement is a regular graph of degree one, namely, a matching of a complete graph on $2 j$ vertices. This establishes $G \simeq \mathscr{C}$, where $\mathscr{C}$ is the graph described earlier for the case $\Delta=2 j-1$.

3.2. Unique Graphs with Maximum Number of Edges for a Given Maximum Degree and Matching Size. We emphasize that graphs considered in this discussion have no isolated vertex. Note that a method is provided in [5] to construct a graph $G$ such that $|E(G)|$ attains the edge bound given by (1). We find the condition when the graphs that attain the maximum edge bound (1) are unique up to isomorphism.

Proposition 10. Let $\mathscr{C}$ be the graph constructed in the Section 3.1 and $G$ be a simple graph with $v:=v(G)$ and $\Delta:=\Delta(G)$ such that $\Delta \geq 2$. If $\lceil\Delta / 2\rceil$ divides $v$ and $|E(G)|=$ $\Delta v+(v\lfloor\Delta / 2\rfloor /\lceil\Delta / 2\rceil)$, then
(a) $v(G \backslash x)=v(G)$ for all $x \in V(G)$,
(b) if $D$ is a component of $G$ with $v(D)>v(\mathscr{C})$, then $(|E(D)| / v(D))<(|E(\mathscr{C})| / v(\mathscr{C}))$,
(c) if $D$ is a component of $G$ with $v(D)<v(\mathscr{C})$, then $(|E(D)| / v(D))<(|E(\mathscr{C})| / v(\mathscr{C}))$,
(d) every component of $G$ is isomorphic to $\mathscr{C}$,
(e) $G$ is unique up to isomorphism.

Proof. Let $G$ be a graph satisfying the conditions of the proposition.

Proof of (a). If the statement (a) is false then there exists a vertex $v \in V(G)$ such that $v(G \backslash v)<v(G)$. This implies

$$
\begin{aligned}
\Delta v+\frac{v}{\lceil\Delta / 2\rceil}\left\lfloor\frac{\Delta}{2}\right\rfloor & =|E(G)| \leq \operatorname{deg}_{G}(v)+|E(G \backslash v)| \\
\leq & \Delta+\Delta(G \backslash v)(v-1) \\
& \left.+\left\lfloor\frac{v-1}{\lceil\Delta(G \backslash v) / 2\rceil}\right\rfloor \mid \frac{\Delta(G \backslash v)}{2}\right\rfloor .
\end{aligned}
$$

We again note that the edge bound, that is, (1) is a nondecreasing function of $\Delta$ for a fixed $v$. Also $\Delta \geq \Delta(G \backslash v)$. Therefore,

$$
\begin{aligned}
& \Longrightarrow \frac{v}{\lceil\Delta / 2\rceil}\left\lfloor\frac{\Delta}{2}\right\rfloor \leq\left\lfloor\frac{v-1}{\lceil\Delta / 2\rceil}\right\rfloor\left\lfloor\frac{\Delta}{2}\right\rfloor \leq \frac{(v-1)}{\lceil\Delta / 2\rceil}\left\lfloor\frac{\Delta}{2}\right\rfloor \\
& \Longrightarrow v \leq v-1 .
\end{aligned}
$$

This contradiction proves (a).

We recall that the graph $\mathscr{C}$ is a factor critical graph with $\Delta\lceil\Delta / 2\rceil+\lfloor\Delta / 2\rfloor$ edges and maximum matching size $\lceil\Delta / 2\rceil$.

Proof of $(b)$. Let $D$ be a component of $G$. Gallai's lemma and (a) imply that $D$ is factor-critical, hence $|V(D)|=2 v(D)+1$ and

$$
|E(D)| \leq\left\lfloor\frac{(2 v(D)+1) \Delta}{2}\right\rfloor=v(D) \Delta+\left\lfloor\frac{\Delta}{2}\right\rfloor .
$$


Note that in the following inequality we again used the fact that the edge bound, that is, (1) is a nondecreasing function of $\Delta$ for a fixed $v$. If $v(D)>v(\mathscr{C})$, then

$$
\begin{aligned}
\frac{|E(D)|}{v(D)} & \leq \Delta+\frac{\lfloor\Delta / 2\rfloor}{v(D)}<\Delta+\frac{\lfloor\Delta / 2\rfloor}{v(\mathscr{C})} \\
& =\Delta+\frac{\lfloor\Delta / 2\rfloor}{\lceil\Delta / 2\rceil}=\frac{|E(\mathscr{C})|}{v(\mathscr{C})} .
\end{aligned}
$$

This proves (b).

Proof of $(c)$. Let $D$ be a component of $G$. By Gallai's lemma and (a), $D$ is a factor-critical graph and hence $|V(D)|=$ $2 v(D)+1$. Thus

$$
|E(D)| \leq \frac{(2 v(D)+1) 2 v(D)}{2}=(2 v(D)+1) v(D) .
$$

Now since $v(D) \leq v(\mathscr{C})-1$, we have

$$
\begin{aligned}
\frac{|E(D)|}{v(D)} & \leq 2 v(D)+1 \leq 2 v(\mathscr{C})-1 \\
& =2\left\lceil\frac{\Delta}{2}\right\rceil-1<\Delta+\frac{\lfloor\Delta / 2\rfloor}{\lceil\Delta / 2\rceil}=\frac{|E(\mathscr{C})|}{v(\mathscr{C})},
\end{aligned}
$$

which proves (c).

Proof of $(d)$. From (b) and (c), every component $D$ of $G$ has $v(D)=v(\mathscr{C})=\lceil\Delta / 2\rceil$ and also $|E(D)|=\Delta\lceil\Delta / 2\rceil+\lfloor\Delta / 2\rfloor$. Since $\mathscr{C}$ is the only graph with maximum matching size $\lceil\Delta / 2\rceil$ and the number of edges $\Delta\lceil\Delta / 2\rceil+\lfloor\Delta / 2\rfloor, D$ must be isomorphic to $\mathscr{C}$. This proves (d).

Proof of (e). This follows from (d).

Now we explore the inverse of Proposition 10. Recall the connected graph $\mathscr{C}$ described in Section 3.1 is the unique graph satisfying (4).

Proposition 11. Let $G_{1}$ be a simple graph such that $\left|E\left(G_{1}\right)\right|=$ $\Delta\left(G_{1}\right) v\left(G_{1}\right)+\left\lfloor v\left(G_{1}\right) /\left\lceil\Delta\left(G_{1}\right) / 2\right\rceil\right\rfloor\left\lfloor\left(G_{1}\right) / 2\right\rfloor$, that is, $G_{1}$ attains the maximum edge bound given by the inequality (1). If $v\left(G_{1}\right) \geq 2, \Delta\left(G_{1}\right) \geq 2$ and $\left\lceil\Delta\left(G_{1}\right) / 2\right\rceil$ does not divide $v\left(G_{1}\right)$, then there exists a simple graph $G_{2}$ such that $\left|E\left(G_{2}\right)\right|=$ $\left|E\left(G_{1}\right)\right|, v\left(G_{2}\right)=v\left(G_{1}\right), \Delta\left(G_{2}\right)=\Delta\left(G_{1}\right)$ and $G_{2}$ is not isomorphic to $G_{1}$.

Proof. We use the method given in [5] to construct a simple graph $G$ such that $|E(G)|=\left|E\left(G_{1}\right)\right|, v(G)=$ $v\left(G_{1}\right)$, and $\Delta(G)=\Delta\left(G_{1}\right)$. Let $G$ have $t:=v\left(G_{1}\right)-$ $\left(\left\lceil\Delta\left(G_{1}\right) / 2\right\rceil\right)\left\lfloor v\left(G_{1}\right) /\left\lceil\Delta\left(G_{1}\right) / 2\right\rceil\right\rfloor$ components isomorphic to $K_{1, \Delta\left(G_{1}\right)}$ and $\left\lfloor v\left(G_{1}\right) /\left\lceil\Delta\left(G_{1}\right) / 2\right\rceil\right\rfloor$ components isomorphic to $\mathscr{C}$ (described in Section 3.1). Let $G_{2}=G$ if $G_{1}$ is not isomorphic to $G$. So assume that $G_{1}$ is isomorphic to $G$. Note that $t:=v\left(G_{1}\right)-\left(\left\lceil\Delta\left(G_{1}\right) / 2\right\rceil\right)\left\lfloor v\left(G_{1}\right) /\left\lceil\Delta\left(G_{1}\right) / 2\right\rceil\right\rfloor \geq 1$ as $\left\lceil\Delta\left(G_{1}\right) / 2\right\rceil$ does not divide $v\left(G_{1}\right)$. If $t \geq 2$ then let $H_{1}$ and $H_{2}$ be two components of $G_{1}$ isomorphic to $K_{1, \Delta\left(G_{1}\right)}$. We remove an edge of $H_{1}$ and then connect the vertex of degree $\Delta\left(G_{1}\right)-1$ of $H_{1}$ to any vertex of degree one in $H_{2}$ by a new edge. Thus, a new graph $G_{2}$ is obtained. It is obvious by construction that $G_{2}$ satisfies hypothesis of Proposition 11 . So, we need to consider only the case $t=1$ to complete the proof. As $v\left(G_{1}\right) \geq 2$ and $t=1, G_{1}$ has at least two components. Hence $G_{1}$ has a component isomorphic to $K_{1, \Delta\left(G_{1}\right)}$ and at least one component isomorphic to $\mathscr{C}$. As $\Delta\left(G_{1}\right)+|E(\mathscr{C})|=$ $\Delta\left(G_{1}\right)+\Delta\left(G_{1}\right)\left(\left\lceil\Delta\left(G_{1}\right) / 2\right\rceil\right)+\left\lfloor\Delta\left(G_{1}\right) / 2\right\rfloor \leq\left\lfloor\left(2\left(\left\lceil\Delta\left(G_{1}\right) / 2\right\rceil+\right.\right.\right.$ $1)+1) \Delta\left(G_{1}\right) / 2$ ], we can coalesce the two components to form a factor-critical component with $2\left(\left\lceil\Delta\left(G_{1}\right) / 2\right\rceil+1\right)+1$ vertices and maximum degree at most $\Delta\left(G_{1}\right)$ which has number of edges equal to $\Delta\left(G_{1}\right)+|E(\mathscr{C})|$ and maximum matching size equal to $v(\mathscr{C})+1$. Thus, again a new graph $G_{2}$ is obtained. It is obvious by construction that $G_{2}$ satisfies hypothesis of Proposition 11.

We can combine the above two propositions in the following theorem.

Theorem 12. Let $G$ be a simple graph such that $v(G) \geq$ 2, $\Delta(G) \geq 2$ and $|E(G)|=\Delta(G) v(G)+\lfloor v(G) /\lceil\Delta(G) /$ $2\rceil\rfloor\lfloor\Delta(G) / 2\rfloor$. $G$ is a unique graph up to isomorphism if and only if $\lceil\Delta(G) / 2\rceil$ divides $v(G)$.

Proof. The above conclusion follows by Propositions 10 and 11.

\section{Graphs Whose Edge Set Can Be Partitioned into Maximum Matchings}

Definition 13. A simple graph $G$ is called friendly-edgecolorable if and only if $E(G)$ has a partition into maximum matchings.

Examples: $C_{4}, C_{2 m}$ for $m \geq 2, K_{2 m}$ for $m \geq 1, K_{2 m+1}$ for $m \geq 1$ and $K_{1, n}$ for $n \geq 1$.

We observe that a proper edge coloring of $G$ is equivalent to partitioning $E(G)$ into matchings (may not be maximum).

Proposition 14. $G$ is a friendly-edge-colorable graph if and only if $|E(G)|=\chi^{\prime}(G) v(G)$.

Proof. We consider a minimal proper edge coloring of $G$. Since every color class of $G$ is a matching and a matching in $G$ can be of size at most $v(G)$, we have $|E(G)| \leq \chi^{\prime}(G) v(G)$. So if $G$ is a friendly-edge-colorable graph, then there is a partition of $E(G)$ into maximum matchings. Hence there is a positive integer $n$ such that $|E(G)|=n v(G)$. This partition corresponds to a proper edge coloring with $n$ colors. Thus, $\chi^{\prime}(G) \leq n$. Hence, $|E(G)|=n v(G) \geq \chi^{\prime}(G) v(G)$. Thus for a friendly-edge-colorable graph $G$, we have $|E(G)|=$ $\chi^{\prime}(G) v(G)$.

Suppose $G$ is not a friendly-edge-colorable graph. We consider an edge coloring of $G$ in $\chi^{\prime}(G)$ colors. Note that each color class is a matching and at least one of the color classes is of size strictly less than $v(G)$ as $G$ is not a friendly-edgecolorable graph. Thus, we get $|E(G)|<\chi^{\prime}(G) v(G)$. 
Proposition 15. If $G$ is a friendly-edge-colorable graph, then any proper edge coloring of $G$ in $\chi^{\prime}(G)$ colors results in a partition of $E(G)$ into maximum matchings.

Proof. Any proper edge coloring of $G$ is a partition of $E(G)$ into matchings (may not be maximum). Consider a proper edge coloring of $G$ in $\chi^{\prime}(G)$ colors. If there is a color class of size strictly less than $v$, then $|E(G)|<\chi^{\prime}(G) v(G)$ which contradicts Proposition 14.

Remark 16. The name "friendly-edge-colorable" is due to Proposition 15 which states that when the least number of colors are used to properly color the edges, the colors are equally distributed so that each color class gets the maximum!

The following theorem characterizes friendly-edgecolorable graphs in Class II.

Theorem 17. Let $G$ be a friendly-edge-colorable graph of Class II such that $\Delta(G) \geq 2$ and $v(G) \geq 2$. Then

(a) $\Delta(G)$ is even,

(b) $\Delta(G) / 2$ divides $v(G)$,

(c) every component of $G$ is isomorphic to $K_{\Delta(G)+1}$, the complete graph of order $\Delta(G)+1$.

Proof. Let $\Delta=\Delta(G)$ and $v=v(G)$. By Proposition 15, $|E(G)|=v(\Delta+1)$. Also by inequality (1),

$$
\begin{aligned}
(\Delta+1) v=|E(G)| & \left.\leq \Delta v+\left\lfloor\frac{v}{\lceil\Delta / 2\rceil}\right\rfloor \frac{\Delta}{2}\right\rfloor, \\
& \leq v \Delta+\frac{v}{\lceil\Delta / 2\rceil}\left\lfloor\frac{\Delta}{2}\right\rfloor \\
& \leq v \Delta+v, \quad\left[\text { as } \frac{\lfloor\Delta / 2\rfloor}{\lceil\Delta / 2\rceil} \leq 1\right] .
\end{aligned}
$$

Thus, $\lfloor v /\lceil\Delta / 2\rceil\rfloor=v /\lceil\Delta / 2\rceil$, which proves (b). Also, $\lfloor\Delta / 2\rfloor /$ $\lceil\Delta / 2\rceil=1$ that implies (a). By Proposition 10, we know that every component of $G$ is isomorphic to $K_{\Delta+1}$, the complete graph of order $\Delta+1$.

Now we will consider friendly-edge-colorable graphs that are in Class I, that is, graphs with edge chromatic index $\Delta$. Next we prove three lemmas that help us characterize friendly-edge-colorable graphs in Class I.

Lemma 18. Let $G$ be a friendly-edge-colorable graph. If there exists a vertex $x \in V(G)$ such that $v(G \backslash x)<v(G)$ then $\operatorname{deg}_{G}(x)=\chi^{\prime}(G)$.

Proof. As $G$ is friendly-edge-colorable, there exists a partition of $E(G)$ into maximum matchings and, by Proposition 14, $|E(G)| / \nu(G)=\chi^{\prime}(G)$. Hence each class, that is, each part of this partition, has size $v(G)$ and there are $\chi^{\prime}(G)$ parts. We denote each color class (i.e., a part in the partion) by $C_{i}$ for all $i \in\left\{1, \ldots, \chi^{\prime}(G)\right\}$. If $\operatorname{deg}_{G}(x)<\chi^{\prime}(G)$ then there is a color missing at $x$. Without loss of generality, let the missing color be 1 . Hence the whole color class $C_{1}$ belongs to $E(G \backslash x)$, that is, $C_{1} \subseteq E(G \backslash x)$. Therefore, $v(G \backslash x) \geq\left|C_{1}\right|$. But $C_{1}$ is a maximum matching of $G$ hence $\left|C_{1}\right|=v(G)$. This contradicts that $v(G \backslash x)<v(G)$. Hence $\operatorname{deg}_{G}(x) \geq \chi^{\prime}(G)$. Also $\operatorname{deg}_{G}(x) \leq \Delta(G) \leq \chi^{\prime}(G)$ by the definition of $\chi^{\prime}(G)$.

Remark 19. Lemma 18 implies that if $G$ is friendly-edgecolorable and there exists $x \in V(G)$ such that $v(G \backslash x)<v(G)$, then $\operatorname{deg}_{G}(x)=\Delta(G)=\chi^{\prime}(G)$ as $\Delta(G) \leq \chi^{\prime}(G)$. Hence $G$ is in Class I. So the contrapositive implies that if $G$ is friendlyedge-colorable and in Class II, that is, not in Class I, then $v(G \backslash x)=v(G)$ for all $x \in V(G)$. By Gallai's lemma, each component of $G$ is a factor-critical component as we noticed in Theorem 17.

Lemma 20. Let $G$ be a friendly-edge-colorable graph. If there exists $x \in V(G)$ such that $v(G \backslash x)<v(G)$ and $|E(G \backslash x)| \geq 1$, then $G \backslash x$ is a friendly-edge-colorable graph and $\chi^{\prime}(G \backslash x)=$ $\chi^{\prime}(G)$.

Proof. As $G$ is friendly-edge-colorable, $E(G)$ has a partition into maximum matchings. Define a coloring, $C$, corresponding to such a partition. Then each color class has size $v(G)$ and there are $|E(G)| / \nu(G)=\chi^{\prime}(G)$ color classes by Proposition 14. Now consider the restriction of $C$ on $E(G \backslash x)$. By Lemma $18, \operatorname{deg}_{G}(x)=\chi^{\prime}(G)$ hence $C_{E(G \backslash x)}$ has $\chi^{\prime}(G)$ color classes each of size $v(G)-1$. As by assumption $v(G \backslash x)=v(G)-1$, $E(G \backslash x)$ has a partition into maximum matchings, implying that $G \backslash x$ is friendly-edge-colorable. Since $G \backslash x$ is friendlyedge-colorable, by Proposition 14, we get

$$
\begin{aligned}
\chi^{\prime}(G \backslash x) & =\frac{|E(G \backslash x)|}{v(G \backslash x)} \\
& =\frac{|E(G)|-\operatorname{deg}_{G}(x)}{v(G)-1} \\
& =\frac{|E(G)|-\chi^{\prime}(G)}{v(G)-1} \\
& =\frac{v(G) \chi^{\prime}(G)-\chi^{\prime}(G)}{v(G)-1} \\
& =\chi^{\prime}(G) .
\end{aligned}
$$

By a nontrivial component of a simple graph, we shall mean a component that has at least an edge, that is, $K_{2}$ is a subgraph of the component.

Lemma 21. G is a friendly-edge-colorable graph if and only if each nontrivial component $C$ of $G$ is friendly-edge-colorable and $\chi^{\prime}(G)=\chi^{\prime}(C)$.

Proof. We first show the if part. Consider a proper edge coloring for each component in $\chi^{\prime}(G)$ colors. This gives the desired partition of the edge set of $G$.

Next we show the only if part. Since $G$ is friendlyedge-colorable, there is a partition of $E(G)$ into maximum 
matchings that has $\chi^{\prime}(G)$ classes (by Proposition 14). We claim that restricting this partition to any component of $G$ gives a partition of the edge set of the component into maximum matchings of the component. Suppose on the contrary that some matching $\mathscr{M}_{\mathscr{C}}$ obtained this way (by restricting a maximum matching $\mathscr{M}$ to $\mathscr{C}$ ) for a component $\mathscr{C}$ is not a maximum matching in $\mathscr{C}$. Then there is an augmenting path relative to this matching in the component. But then this is an augmenting path, relative to the corresponding matching $\mathscr{M}$ of $G$, in $G$. Hence $\mathscr{M}$ is not a maximum matching of $G$. This contradicts the fact that $\mathscr{M}$ is a maximum matching.

If for some component $C, \chi^{\prime}(C) \neq \chi^{\prime}(G)$ then $\chi^{\prime}(C)<$ $\chi^{\prime}(G)$ (as $\chi^{\prime}(C) \leq \chi^{\prime}(G)$ for $C$ is a component of $G$ ). Now consider any proper edge coloring of $G$ in $\chi^{\prime}(G)$ colors and for each component order color classes greedily. For each $1 \leq i \leq \chi^{\prime}(G)$, we denote the $i$ th color class by $\mathscr{D}(i)$. Then the first $\chi^{\prime}(\mathscr{C})$ color classes will have strictly larger size than the remaining color classes $\left\{\mathscr{D}\left(\chi^{\prime}(\mathscr{C})+1\right), \mathscr{D}\left(\chi^{\prime}(\mathscr{C})+\right.\right.$ $\left.2), \ldots, \mathscr{D}\left(\chi^{\prime}(G)\right)\right\}$. This contradicts that each color class is of the same size $v(G)$ as shown by Proposition 15 .

Remark 22. In the previous lemma the condition that $\chi^{\prime}(G)=$ $\chi^{\prime}(C)$ for all components $C$ is necessary otherwise counter examples exist. For instance, let $G$ be the graph consisting of two components one of which is $K_{3}$ and the other one is $K_{1,4}$.

Theorem 23. If $G$ is a nontrivial, Class I, friendly-edgecolorable graph then $E(G)$ has a partition into the following two kinds of subgraphs:

(i) $K_{1, \Delta(G)}$

(ii) factor critical, friendly-edge-colorable graphs with edge chromatic index $\chi^{\prime}(G)$.

Proof. Let $G_{0}:=G$. If there exists $x_{1} \in V(G)$ such that $v\left(G \backslash x_{1}\right)<v(G)$ then remove all edges incident to $x_{1}$ and define $G_{1}:=G \backslash x_{1}$. Since by Lemma 18 and Remark $19 \operatorname{deg}_{G}\left(x_{1}\right)=\Delta$, we removed a $K_{1, \Delta(G)}$ from $G$. Note that $G_{1}$ is a friendly-edge -colorable graph and $\chi^{\prime}\left(G_{1}\right)=\chi^{\prime}(G)$ by Lemma 20. By Lemma 21, each component of $G_{1}$ is also a friendly-edge-colorable graph and has edge chromatic index $\chi^{\prime}(G)$. Similarly, for $i \geq 1$ define $G_{i}:=G_{i-1} \backslash x_{i}$ if there exists $x_{i} \in V\left(G_{i-1}\right)$ such that $v\left(G_{i-1} \backslash x_{i}\right)<v\left(G_{i-1}\right)$. We remove the vertices $x_{i}$ and all edges adjacent to each of these vertices. Corresponding to each of the $x_{i}$ 's, we get a subgraph isomorphic to $K_{1, \Delta(G)}$. For some large enough $i$, we have only those nontrivial components $C$ in $G_{i}$ such that $v(C \backslash x)=$ $v(C)$ for all $x \in V(C)$. By Gallai's lemma, these are the factor critical components. Also by Lemma $21, \chi^{\prime}(C)=\chi^{\prime}\left(G_{i}\right)=$ $\chi^{\prime}(G)$.

Remark 24. Reader can review Figure 1 and notice that the degree of the vertex $x$ in Figure 1 is 3 and $x$ must be covered by every maximum matching. Also, removal of the vertex $x$ from the graph yields a graph whose only nontrivial component is factor-critical.

\section{Acknowledgments}

The author would like to thank Dr. Nishali Mehta, Dr. Naushad Puliyambalath, and Professor Ákos Seress for their valuable comments and help to improve the paper.

\section{References}

[1] I. Holyer, "The NP-completeness of edge-coloring," SIAM Journal on Computing, vol. 10, Article ID 718720, 1981.

[2] D. P. Sanders and Y. Zhao, "Planar graphs of maximum degree seven are class I," Journal of Combinatorial Theory Series B, vol. 83, no. 2, pp. 201-212, 2001.

[3] D. B. West, Introduction toGraph Theory, Prentice Hall, 1996.

[4] P. Erdos and R. J. Wilson, "On the chromatic index of almost all graphs," Journal of Combinatorial Theory Series B, vol. 23, no. 2-3, pp. 255-257, 1977.

[5] N. Balachandran and N. Khare, "Graphs with restricted valency and matching number," Discrete Mathematics, vol. 309, no. 12, pp. 4176-4180, 2009.

[6] V. Chvátal and D. Hanson, "Degrees and matchings," Journal of Combinatorial Theory Series B, vol. 20, no. 2, pp. 128-138, 1976.

[7] L. Lovasz and M. D. Plummer, Matching Theory, North Holland, Amsterdam, The Netherlands, 1986. 


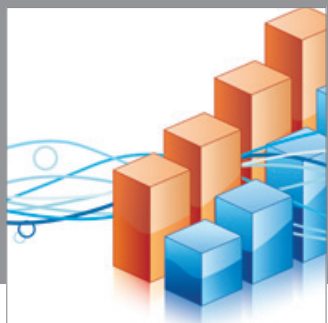

Advances in

Operations Research

mansans

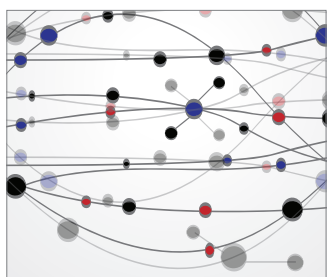

The Scientific World Journal
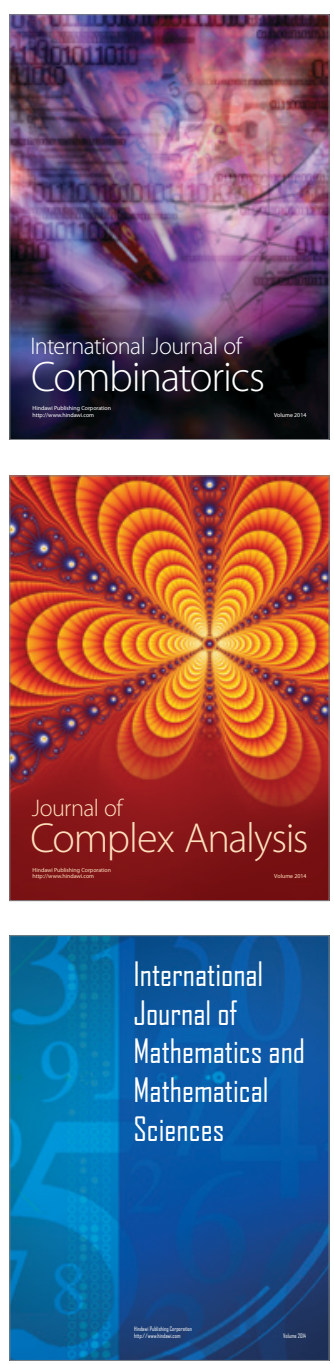
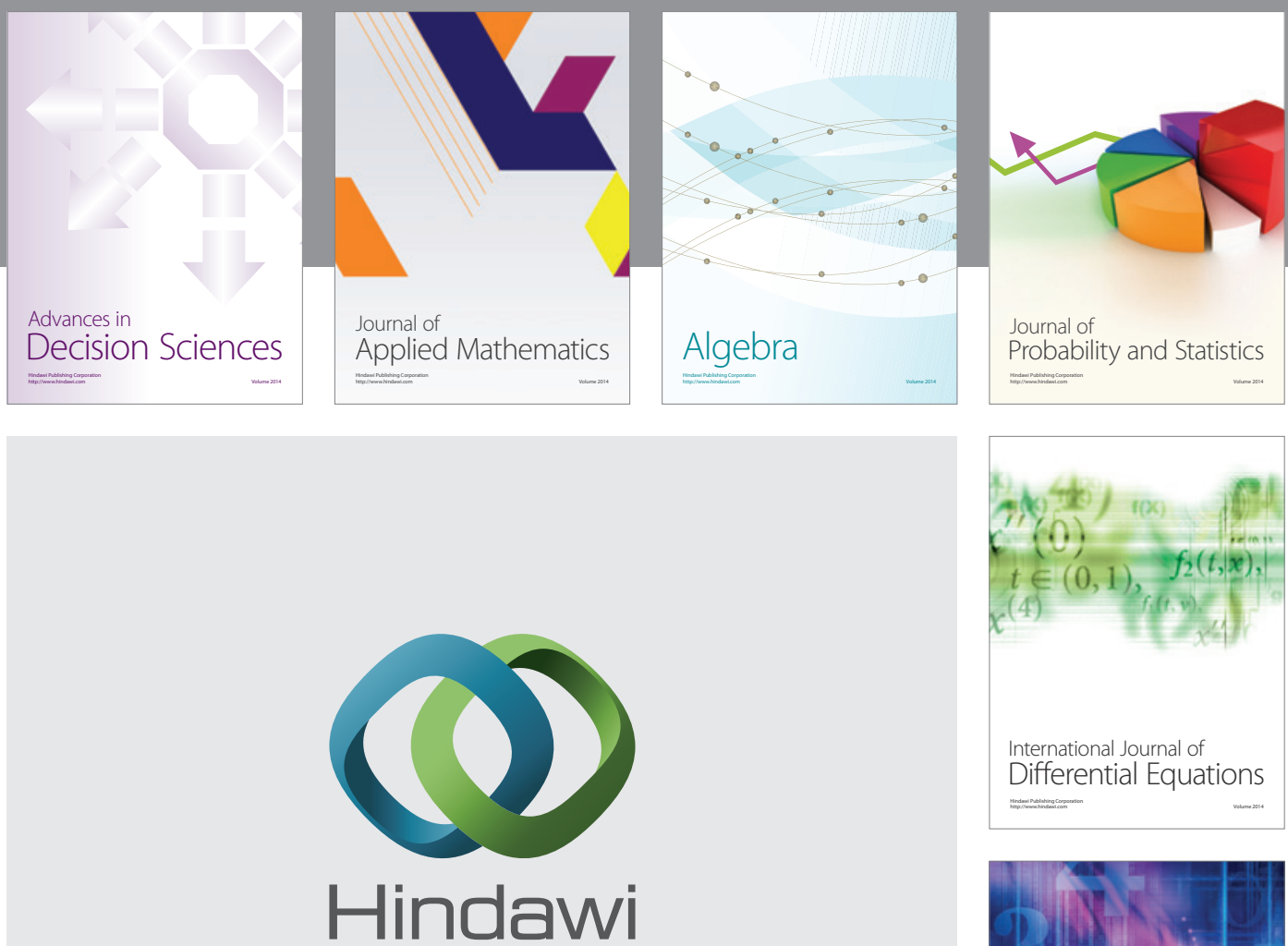

Submit your manuscripts at http://www.hindawi.com
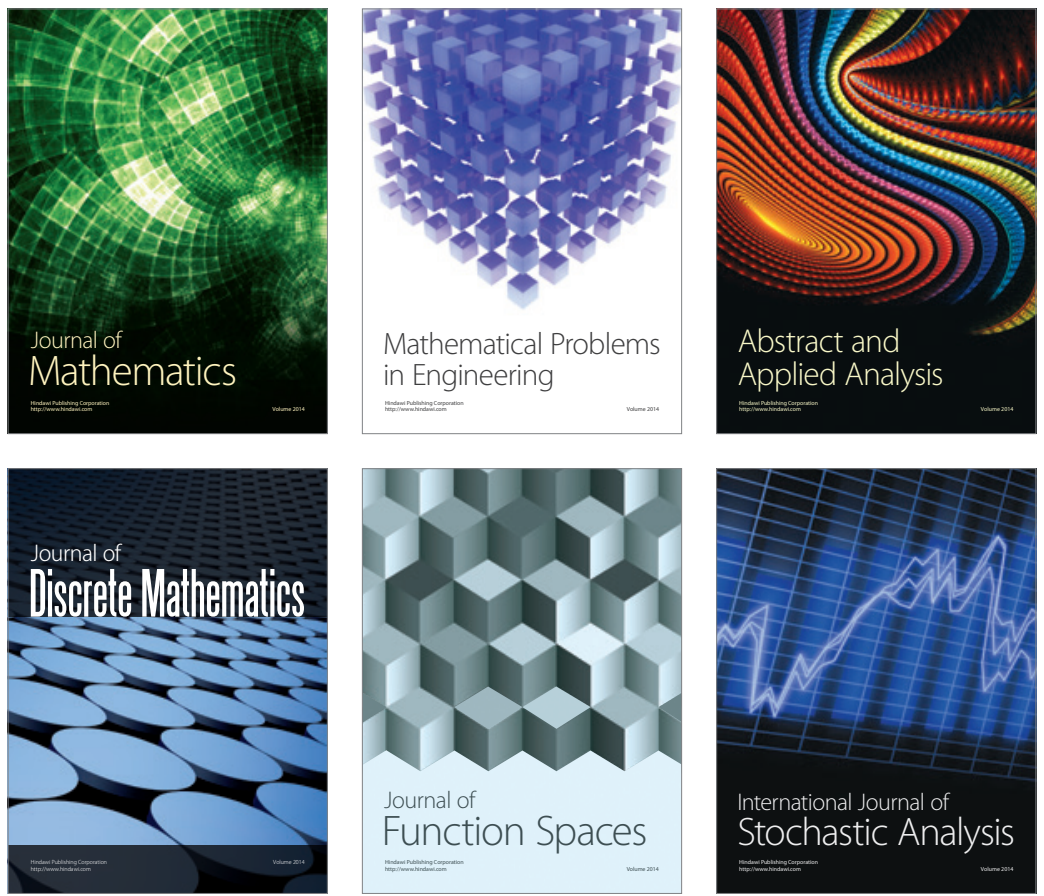

Journal of

Function Spaces

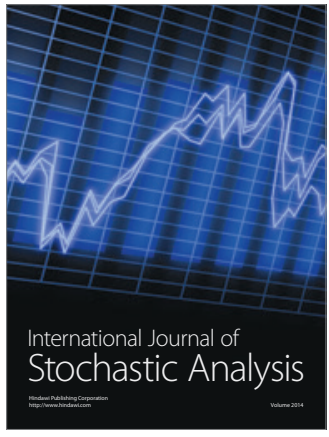

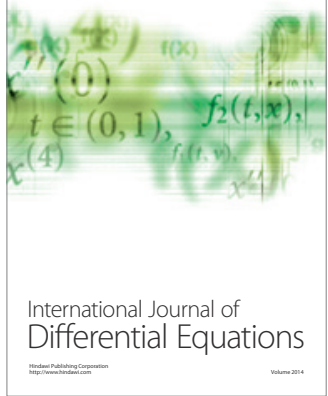
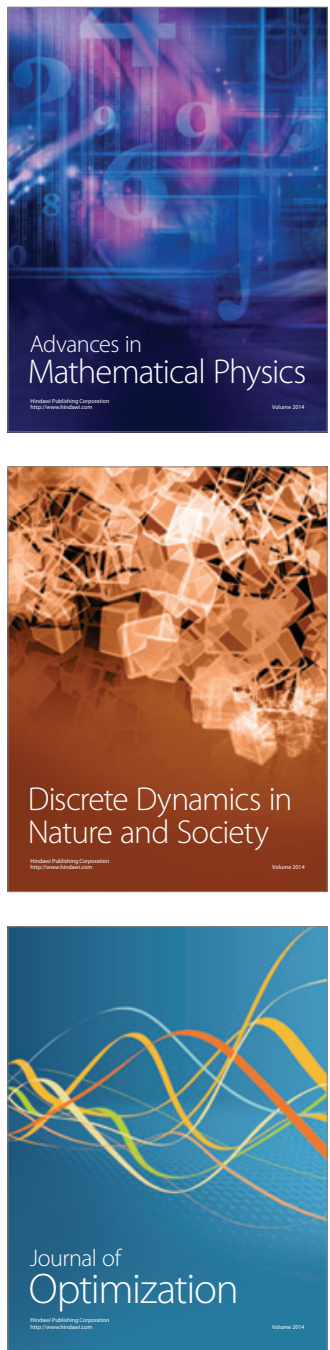